\title{
Micro-electromechanical film bulk acoustic sensor for plasma and whole blood coagulation monitoring
}

Da Chen ${ }^{1,2, *}$, Shuren Song ${ }^{2}$, Jilong $\mathrm{Ma}^{2}$, Zhen Zhang ${ }^{2}$, Peng Wang ${ }^{2}$, Weihui Liu ${ }^{2}$, Qiuquan $\mathrm{Guo}^{3, *}$

1 State Key Laboratory of Mining Disaster Prevention and Control Co-founded by Shandong Province and the Ministry of Science and Technology, Shandong University of Science and Technology, Qingdao 266590, China

2 College of Electronics, Communications, and Physics, Shandong University of Science and Technology, Qingdao 266590 PR China

3 Mechanical \& Materials Engineering, Western University, London, Ontario N6A 3K7, Canada

* Corresponding author: Da Chen, Qiuquan Guo

E-mail: phychenda@163.com; qguo29@uwo.ca

Phone/fax: +86-053286057555; 1-5194321167

$-1-$ 


\begin{abstract}
Monitoring blood coagulation is an important issue in the surgeries and the treatment of cardiovascular diseases. In this work, we reported a novel strategy for the blood coagulation monitoring based on a micro-electromechanical film bulk acoustic resonator. The resonator was excited by a lateral electric field and operated under the shear mode with a frequency of $1.9 \mathrm{GHz}$. According to the apparent step-ladder curves of the frequency response to the change of blood viscoelasticity, the coagulation time (prothrombin time) and the coagulation kinetics were measured with the sample consumption of only $1 \mu \mathrm{l}$. The procoagulant activity of thromboplastin and the anticoagulant effect of heparin on the blood coagulation process were illustrated exemplarily. The measured prothrombin times showed a good linear correlation with $R^{2}=0.99969$ and a consistency with the coefficient of variation less than $5 \%$ compared with the commercial coagulometer. The proposed film bulk acoustic sensor, which has the advantages of small size, light weight, low cost, simple operation and little sample consumption, is a promising device for miniaturized, online and automated analytical system for routine diagnostics of hemostatic status and personal health monitoring.
\end{abstract}

\title{
Keywords:
}

Acoustic resonator; film bulk acoustic resonator; blood coagulation; micro-electromechanical system; viscosity sensor 


\section{Introduction}

Monitoring of blood coagulation is an integral part in the treatment of patients with cardiovascular problems and surgeries when blood gets into contact with artificial surface. For instance, in the surgical procedures employing cardiopulmonary bypass as well as for patients requiring dialysis treatment, extracorporeal circulation of blood is regularly used in conjunction with anticoagulants. These treatments make it necessary to frequently monitor the blood coagulation parameters to keep a sufficient level of anticoagulation in order to decrease the risk of excessive bleeding. In addition, the oral anticoagulant drugs are widely used for the therapy atrial fibrillation, pulmonary embolism, phlebothrombosis and other diseases. For the patients, it is very important to keep the blood coagulation parameters on an appropriate level in daily life to avoid the risk of thrombus or bleeding. In the hospitals, the coagulation parameters in different assays are usually measured by thromboelastography (TEG), paramagnetic particle methods or optical methods (Harris et al. 2013). Although these technologies can offer precise and standardized results, they require complicated instrumentations and professional operators. Moreover, the analytical devices currently on the market necessitate the manual transfer of blood samples from the patient to separate devices and can not permit continuous sampling and real-time measuring. Therefore, there is an urgent need for the miniaturized, online and automated analytical system for the routine diagnostics of hemostatic status and personal health monitoring.

Acoustic biosensors, represented by a quartz crystal microbalance (QCM), have been 
used to measure the viscosity change during the coagulation process (Duner et al. 2013; Efremov et al. 2013; Hussain et al. 2015; Jin et al. 2013; Müller et al. 2010). However, the typical QCM device has the size of dozens of square millimeter and the frequency of several megahertz (Beckera and Cooper 2011). Further miniaturization of QCM devices are limited by the capability of traditional mechanical cutting and assembling method. Over the past decade, micro- and nanomechanical sensors, represented by microcantilevers, have been developed for the environment, chemical, and biological detections (Anja et al. 2011; Eom et al. 2011; Waggoner and Craighead 2007). The mass resolutions were achieved to zeptogram and nanogram when the nanomechanical resonators operated in vacuum and fluid environment, respectively (Arlett et al. 2011). Recently, film bulk acoustic resonator (FBAR) has become another promising micro-electromechanical candidate as an alternative to QCM for the gas detection (Chen et al. 2011b; Lu et al. 2015) and bio-sensing applications (Pang et al. 2012; Wang et al. 2014). FBAR features a 1-2 micron-thick piezoelectric thin film, such as AlN (Wingqvist 2010), ZnO (Flewitt et al. 2015), $\mathrm{Pb}(\mathrm{Zr}, \mathrm{Ti}) \mathrm{O}_{3}$ (Zinck et al. 2005), $\mathrm{Ba}_{\mathrm{x}} \mathrm{Sr}_{1-\mathrm{x}} \mathrm{TiO}_{3}$ (Lee and Mortazawi 2016), and allows a significantly higher frequency and sensitivity than those of QCMs (Voiculescu and Nordin 2012). As an electroacoustic device, the resonant states of FBAR can be affected by the damping of adjacent medium when the device works in the liquid environment, which makes it feasible to monitor the viscoelasticity changes during the biological reaction (Chen et al. 2013; Xu et al. 2012). The size of the FBAR device is within $1 \mathrm{~mm}^{2}$, which remarkably reduces the form factor and 
manufacturing cost by the batch fabrication based on micro-electromechanical system (MEMS) technology. Furthermore, the miniaturization of the device can handle an extremely small sample volume and perform high throughput tests for the biochemical sensing applications. The disadvantage of the FBAR device is that the device is hardly utilized repeatedly because the material absorbed on the device surface after several times tests is difficult to be removed. As a result, the FBAR devices are seen as disposable sensors for the biochemical applications (Flewitt et al. 2014).

In this study, we present a lateral field excited FBAR for the monitoring of blood coagulation. A portable testing system consisted of oscillators, frequency signal processing and readout circuits was utilized with the availability of direct digital signals. The sequential coagulation stages and the prothrombin times (PT) of plasma and whole-blood samples were measured through the frequency response. The precision and practicability of the proposed FBAR sensor was evaluated by a commercial coagulometer. The procoagulant activity of thromboplastin and the effect of a typical anticoagulant drug (heparin) on the blood coagulation was exemplarily shown.

\section{Materials and methods}

\subsection{Reagents}

Blood samples were collected from an apparently healthy donor by the finger-stick method at the clinical laboratory of Huangdao district hospital, Qingdao, China. The whole-blood samples were treated with $3.8 \%$ sodium citrate. The plasma samples 
were obtained from the whole blood by centrifugation at $3000 \mathrm{rpm}$ for $10 \mathrm{~min}$. The blood samples were stored at $4{ }^{\circ} \mathrm{C}$ before use. Rabbit brain thromboplastin (TP) was provided by an instrument vendor of coagulometer, Punlong Medical Equipment Co., LTD (Nanjing, China). The TP concentration used in the experiments was given in arbitrary units per $\mathrm{ml}$ (unit/ml); one unit/ml corresponds to a 1000-fold dilution of the pooled TP. $\mathrm{CaCl}_{2}$ and heparin solution were purchased from Sinopharm Chemical Reagent Co., LTD (Shanghai, China). All reagents were stored refrigerated before use.

\subsection{The configuration of the FBAR sensor}

Fig. 1 shows the lateral field excited FBAR sensor and the testing system for the monitoring of blood coagulation. The sensor was fabricated using a standard MEMS process. As shown in Fig. 1 (a), A 1.5- $\mu$ m-thick piezoelectric AlN film was built on an acoustic Bragg reflector consisting of three-period alternating layers of $\mathrm{SiO}_{2}$ and $\mathrm{W}$ with the thickness of one quarter wavelength $(0.46$ and $0.35 \mu \mathrm{m}$, respectively). The 100-nm-thick Au electrodes deposited on the AlN film were designed to be paralleled with the gap of $5 \mu \mathrm{m}$ to generate a lateral electric field. A stable shear mode resonance was excited by the lateral electric field with the frequency of $1.94 \mathrm{GHz}$ and the $\mathrm{Q}$ factor of 430 in water (Fig. S1). A testing pool with the dimensions of $6 \mathrm{~mm} \times 3 \mathrm{~mm}$ $\times 0.5 \mathrm{~mm}$ was fabricated with the sidewall of PDMS to make the resonator surface have a good contact with the liquids. Instead of using $\mathrm{SiO}_{2} / \mathrm{AlN}$ as the Bragg reflector in the literature (Chen et al. 2011a), it was used $\mathrm{SiO}_{2} / \mathrm{W}$ Bragg reflector due to the smoother surface (decrease of $54 \%$ of the roughness, Fig. S2), which reduced the 
wave scattering and improved the Q factor of the device (from 313 to 430).

Figure 1

\subsection{The portable testing system of the FBAR sensor}

In the testing system as shown in Fig 1 (b) and (c), the differential frequency processing was used to minimize the environmental disturbance. For this purpose, two identical FBAR resonators were assembled on the system as the detector and the reference, respectively. The testing liquid was only loaded on the detector device resulting in a frequency difference between the detector and unloaded reference resonator. The details of the circuit design were given in the Fig. S3. In the output spectrum of the FBAR oscillator (Fig. S4), the amplitude of the resonant peek was $-3.439 \mathrm{dBm}$ at $1.945 \mathrm{GHz}$ and the average frequency noise produced by the oscillator circuit was about $0.2 \mathrm{dBm}$. The frequency stability and resolution are related to the noise level. In the FBAR sensor, the noise mostly derived from the thermal noise, 1/f noise due to the quantum fluctuation and phonon scattering by defects, and the wire bonding noise between the device electrodes and the circuit board (Fcrre-Pikal et al. 2001).

\subsection{The experimental procedures}

At first, the detector and the reference FBAR devices worked at same frequency. For the coagulation monitoring, $10 \mu \mathrm{l}$ citrated plasma or whole-blood samples, $1 \mu \mathrm{l}$ thromboplastin solution and $1 \mu 25 \mathrm{mM} \mathrm{CaCl}_{2}$ were thoroughly mixed. Then, a droplet of $1 \mu$ mixture solution was dripped on the surface of detector device, 
resulting in a change in the resonant frequency. Then, a droplet of $1 \mu 1$ mixture solution was dripped on the surface of detector device, and the reference device with same resonant frequency was kept at an unloaded state. The resonant frequency change of the sample was recorded. The frequency difference of the two devices was used as the response of the testing system. After each coagulation measurement, the device surface was scrubbed using 3\% hydrogen peroxide solution and subsequently $10 \%$ oxalic acid solution. Then the resonant frequency was measured and compared with its intrinsic value to evaluate the reusability of the device (Fig. S5). After four times of measurements, the changes of the resonant frequency were found to be less than $20 \mathrm{kHz}$. These deviations can be ignored because that the total frequency downshift due to blood coagulation was more than $8.5 \mathrm{MHz}$. However, more reused times resulted in an obvious decrease in the resonant frequency due to the unremoved fibrin on the device surface. As a consequence, each FBAR device was used four times in the experiments.

A commercial mechanical coagulometer based on the double magnetic beads method (PUN-2048, Punlong Medical equipment co., LTD, Nanjing, China) was used to evaluate the precision of the proposed sensor. The whole blood, plasma and user's specific samples can be assayed using this coagulometer. The method is immune to the turbidity disturbance and satisfies the requirements of the various test conditions in our experiments. All the experiments were conducted at room temperature $\left(22^{\circ} \mathrm{C}\right)$. The blood samples were restored to room temperature before each measurement to avoid the temperature effect on the viscosity of the blood. 


\section{Results}

\subsection{Viscosity characterization of the FBAR sensor}

The damping effects on the acoustic resonator depend on viscosity and density of the liquids loaded on the surface of the piezoelectric layer. The glycerol solutions are well suitable to characterize the viscous damping effects on the acoustic devices because its density varies hardly with the concentration (Martin et al. 1993). According to the theory proposed by Keiji Kanazawa et al. (Keiji Kanazawa and Gordon Ii 1985), the frequency shift of an acoustic resonator is linearly proportional to the value of $\sqrt{\rho_{l} \eta_{l}}$ in Newtonian liquids as follows:

$$
\Delta f_{N}=-f_{R}^{3 / 2} \sqrt{\frac{\rho_{l} \eta_{l}}{\rho_{0} \mu_{0} \pi}}
$$

where $\Delta f_{N}$ is the frequency shift in the Newtonian liquid; $\rho_{1}$ and $\eta_{1}$ are the mass density and the viscosity of the loading liquids, respectively; $f_{\mathrm{R}}$ is the resonant frequency in air; $\rho_{0}$ and $\mu_{0}$ denote the density and elastic modulus of the piezoelectric layer, respectively.

The aqueous glycerin solutions with the percent weight of $0-90 \%$ were used to characterize the viscosity response of the FBAR sensor as shown in Fig. 2, of which the frequency difference of the reference device and the detector device was used as the response and the error bars are standard deviation (S. D.) of the ten times measurements. In the range of from 1 to $8.64 \mathrm{~kg} \cdot \mathrm{m}^{-2} \mathrm{~s}^{1 / 2}$ for $\sqrt{\rho_{l} \eta_{l}}$ (glycerin concentration 0-80\%), a linear frequency-viscosity dependence with the slope of approximate $337 \mathrm{kHz} \cdot \mathrm{m}^{2} \mathrm{~s}^{1 / 2} / \mathrm{kg}$ was observed, which agrees well with the Newtonian fluid model of the acoustic device. The frequency shift of thickest aqueous glycerin concentration $(90 \%)$ deviates from the linear relationship, suggesting that the high 
viscosity glycerol loaded on the resonator surface can not be considered as a Newtonian liquid and its behavior becomes Maxwelllian viscoelastic (Martin et al. 1993). In this situation, the frequency shift is related to the dissipation $(D)$ and can be simplified to the following (Parlak et al. 2013):

$$
\Delta f_{M}=\Delta f_{N}+\frac{f_{R} \Delta D}{2}
$$

where

$$
\Delta D \approx-2 f_{R}^{1 / 2} \sqrt[2]{\frac{\rho_{l} \eta_{l}}{\rho_{0} \mu_{0} \pi}}
$$

$\Delta D$ is the dissipation change due to the strong fluid effect. In this experiment, the value of $\Delta D$ can be estimated to be $1.2 \times 10^{-3}$ for the $90 \%$ glycerin solutions.

Figure 2

\subsection{The blood coagulation monitoring}

The blood samples were activated by homogenously distributed calcium ions and TP. In the extrinsic pathway of coagulation (Efremov et al. 2013), TP (tissue factor) activates factor VII, which in turn leads to an activation of factor X. Together with activated factor $\mathrm{V}$, calcium ions and phospholipids activated factor $\mathrm{X}$ forms the so-called prothombinase complex. Finally, this complex catalyzes the conversion of prothrombin to thrombin. Thrombin as a central protein in the coagulation cascade cleaves fibrinogen to fibrin. During the polymerization and growth of fibrin network, the blood viscoelasticity changes (Müller et al. 2010).

In this work, five plasma samples containing various levels of concentration of TP (0-100 unit $/ \mathrm{ml})$ were studied. After the samples were separately dropped onto the sensor, the frequencies decreased about $7.5 \mathrm{MHz}$ immediately due to the sudden loading and viscosity changes (Fig. S6). In order to clarify the details of the frequency behavior during the blood coagulation reaction, the time-dependent frequency profiles 
after the plasma and whole-blood samples dropping were shown in Fig. 3. The coagulation responses were similar in the shape to those of QCM (Vikinge et al. 2000b) and contour-mode FBAR (Xu et al. 2012). In the case of no TP, the frequency nearly kept in a constant value over a long time after the sudden frequency falling. In contrast, for both the plasma and whole-blood samples mixed with TP, the frequencies remained almost constant firstly and then gradually dropped to a relatively low value. The initial plateau time and descending time in the frequency responses were extremely dependent on the TP concentration. The more TP contained, the less step-ladder curve resulted owing to the fast coagulation reaction.

Figure 3

The frequency changes revealed the sequential stages of the coagulation reaction. At first, the prothrombin converted into thrombin under the action of thromboplastin. The thrombin as central protein cleaved fibrinogen to fibrin. During this enzyme cascade reaction, the blood viscoelasticity, and thus the resonant frequency, were not changed (Müller et al. 2010). Then, with the polymerization of fibrin, the liquid viscosity increased and more fibrin fibers contacted with the resonator surface, resulting in a continuous decline in the resonant frequency. Finally, a steady state with no further changes reached after the completion of fibrin network.

\subsection{The test of PT values}

The PT value is an important parameter for the activity of coagulation factors in the extrinsic pathway and the most sensitive indicator for the anticoagulant therapy. After the activation of thromboplastin and calcium ions, the PT values of the blood samples can be determined as the end of fibrin polymerization. As a novel method for coagulation analysis, it is essential to choose a characteristic point in the frequency 
response to determine the PT value. For this purpose, as shown in Fig. 3, the first stable frequency after sample dropping (frequency difference $\sim 7.5 \mathrm{MHz}$ ) was defined as the base line to evaluate the decrease of frequency for each TP concentration. The times when the frequency decreased to three typical percentages $(90 \%, 95 \%, 100 \%)$ of the maximum downshift of frequency $(\Delta f)$ were compared with the results acquired by the commercial coagulometer (Tab. S1). The results showed that the times when the frequency decreased to $95 \%$ were nearest to the PT values measured by the coagulometer. Consequently, these characteristic points were determined as the PT values of the blood samples as summarized in Tab. 1. As expected, more TP in the sample caused shorter coagulation time, which were in the range of 130 700 s. The coefficient of variation (C. V.) between the two methods was less than 5\%. In addition, the frequency responses to the same volume of TP solutions without blood were also measured (Fig. S7). It was found that the base frequency line was not affected by the TP concentration.

\section{Table 1}

\subsection{A Comparison with commercial coagulometer}

In order to evaluate the practicability of the proposed biosensor, a commercial coagulometer was used to measure the coagulation time and compare with the FBAR sensor. The correlation and consistency of the two methods were shown in Fig. 4. As shown in Fig. 4 (a), a linear relationship was found between the two methods with the regression equation of $y=0.994 x+3.701\left(R^{2}=0.99969\right)$. In both science and clinical research, a well-established procedure for the comparison of two different 
measurement techniques is Bland-Altman plot. This type of diagram was used to compare our proposed sensor with the commercial coagulometer as shown in Fig. 4 (b). The value differences of the two methods were plotted against the mean of the corresponding values. One can see that all data points lied in the given range of mean \pm 2 S.D. with a symmetrical distribution, which declare that about $95 \%$ of the values were within two standard deviations. The data in the Bland-Altman plot showed that none of the methods delivered higher or lower values. The measurement consistency of the FBAR sensor with the well-approved method illustrated that the proposed sensor can be used as a feasible tool to analyze blood coagulation.

Figure 4

\subsection{Heparin sensitivity test}

In both laboratorial blood testing and clinical therapy, heparin is a widely used anticoagulant drug (Freedman 1992). Heparin binding to thrombin alters the affinity towards fibrinogens, which weakens the fibrin generation and therefore slows down the coagulation process (Kaatz 2010). Here we used the FBAR sensor to demonstrate the anticoagulant effect of the heparin by adding $5 \mu$ heparin solution to the plasma samples (activated by 50 unit/ml TP). Fig. 5 shows the time-dependent resonant frequency profiles at the heparin concentrations of $0.2-1 \mathrm{IU} / \mathrm{ml}$. Obviously, the addition of heparin resulted in an increases of coagulation time, which showed very good accordance with the FBAR sensor and the commercial coagulometer (Fig. S8). In addition, when excess quantity of heparin was added in the blood sample, the both methods showed the absence of coagulation process during the test time. These results clearly indicated the anticoagulant effect of heparin on the blood coagulation. 
Figure 5

\subsection{The kinetics of blood coagulation}

In order to reveal the coagulation kinetics, the time-dependent frequency profile of the FBAR sensor was fitted using a sigmoid curve (Evans et al. 2008; Jain et al. 2016; Vikinge et al. 2000a) as follows (Fig. S9):

$$
f(t)=\frac{\Delta f}{1+e^{-2 k\left(t-t_{\text {tag }}\right)}}
$$

where $f(t)$ is the frequency response at time $t, \Delta f$ is the maximum frequency downshift, $k$ is the reaction rate constant, and $t_{\text {tag }}$ is the time at which the frequency equals the half value of $\Delta f$.

The extracted kinetics parameters were listed in Tab. S2 and Tab. S3. As expected, the blood sample activated by higher concentration TP had a higher reaction rate constant $k$ (Fig. S10 a). According to the gel architecture proposed by Blombäck et al.(Blombäck et al. 1994), the fibrin network structure is determined by the initial coagulation rate, which is related to the concentration of activator and fibrinogen. A higher activator concentration gives a greater number of nucleation sites from which the fibrinogen monomers can start the polymerization, thus giving a higher polymerization rate than that at a lower activator concentration. In addition, the presence of heparin reduced the coagulation time and resulted in a low reaction rate constant (Fig. S10 b).

The blood viscoelastic properties and coagulation kinetics are influenced by the hematocrit, morphology of the red cell and the concentration levels of cholesterol, 
triglyceride and fibrinogen. The diet, habit, health status and usage of medicine have a significant impact on the human blood viscoelasticity and coagulation kinetics. The frequency responses of the FBAR sensor to the blood coagulation are also influenced by these factors. In the further studies, a large sample database need to be established for the new technology.

\section{Discussion}

The uncertainty of the system is closely related to the frequency resolution and the noise of the FBAR sensor. The frequency resolution $R$ was estimated to be about 30 $\mathrm{Hz}$ from the frequency fluctuation at the based resonant frequency $f_{0}$ as follows (Rodriguez-Pardo et al. 2004)

$$
R=\sigma(\tau) \cdot f_{0}
$$

where $\sigma(\tau)$ is the Allan variance based upon the resonant frequency measurements over time (Fig. S11).

In the realized testing system, two identical FBAR devices were used. One measured the change of blood viscoelasticity, while the other one detected the background resonance that were subtracted to provide a better signal. In consideration of the requirement of linear output, the minimum detectable frequency of the Barron transformer was designed to $20 \mathrm{kHz}$, which is enough to distinguish the frequency changes due to the blood coagulation. As shown in the time-dependent frequency profiles (Fig. 3), the average noise level and the signal-to-noise ratio were estimated to be $36 \mathrm{kHz}$ and 208 , respectively.

According to theoretical model of acoustic resonator, the shear wave penetration 
depth is $200-300 \mathrm{~nm}$ in the blood (Keiji Kanazawa and Gordon Ii 1985). That is to say that the sample volume suffered the shear perturbations is very small; much smaller than, for example, the typical size of red cell. The oscillations occurring close to the surface cannot actually perturb blood cells until they are attached (Efremov et al. 2013). Furthermore, the blood proteins, especially fibrinogen and fibrin fibers, behave not viscously but rigidly after their attachment for a wide range of coating materials (Andersson et al. 2005). When the blood start to clot, not only the change in viscosity but also the change in elasticity and the fibrin adhesion may occur simultaneously as the result of the formation of fibrin fibers (Lakshmanan et al. 2014). In addition, other factors, such as the nonhomogeneous shear vibration, boundary effect and micro-form standing wave for the ultra-small sample volume, may influence the resonant state of the FBAR sensors. Actually, the frequency shifts of the FBAR sensor as shown in Fig. 3 were determined by the comprehensive viscoelasticity changes during the blood coagulation instead of only the absolute viscosity, which limits the application of the FBAR sensors in the measurement of blood viscosity. A detail theoretical model to predict the effects of viscosity and other factors on the FBAR device will be studied in our further work.

As shown in Fig. 3 (a), the maximum frequency downshift $\Delta f$ was observed decreased with the increase of TP concentration. The dependence of the maximum response on the activator concentration was in agreement with the results obtained from QCMs (Chang et al. 2000; Cheng et al. 1998; Vikinge et al. 2000b), SPR (Vikinge et al. 2000a) and the shear gradient-activated microfluidic device (Jain et al. 
2016). This behavior is in accordance with the fact that the fibrin fibers grow thicker and longer as the increase of coagulation time (Efremov et al. 2014). At a constant fibrinogen concentration, the activator concentration determines the number of nucleation sites. The fiber formation from fewer nucleation sites leads to fewer, but thicker, rigid and longer fibers at the low activator concentration (Blombäck et al. 1994). In contrast, the rapid coagulation process, at high activator concentration, leads to many thin and small fibrin fibers that expose a relative larger area to the surrounding liquid and thus are considered more flexible (Efremov et al. 2014). As a consequence, the low-density network results in a less frequency shift at the high TP concentration as shown in Fig. 3 (a), which is also in agreement with the microstructure of the fibrin network observed by Viking et al. (Vikinge et al. 2000a) and Lakshmanan et al.(Lakshmanan et al. 2016). Their results showed that the fibers were shorter, wider and fewer in number as a result of slow formation rate in the presence of low TF concentration while the fibers became longer, thinner and less numerous due to the more rapid onset of clot formation at higher TF concentrations. Moreover, for the whole-blood samples, the frequency behaviors showed no obvious dependence on the TP concentration, although the coagulation times did. The main difference between the whole blood and plasma is the presence of blood platelets. As an important modulators of blood coagulation in vivo, platelets provide activation and nucleation sites for the fibrin polymerization (Cao et al. 2007). Compared to the plasma, the effect of TP on the buildup of fibrin network may be modified due to the incorporation of platelets and other blood cells on the fibrin fibers 
(Wu et al. 2016).

The blood coagulation monitoring device described here provides several potential advantages over other analytical devices. First, the FBAR device can be mass-manufactured on wafer level at low cost utilizing the existing silicon microelectronics process, which is an essential requirement for single-use disposability. As a comparison, the market price of the FBAR filter chip which Avago shipped in 2006 has been reduced to less than 2 dollar in the recent years (Ruby et al. 2012). Another promising advantage is the feasibility of the integration of the FBAR sensor array with the CMOS electronics in a single-chip configuration. For example, Siemens has demonstrated an on-chip array composed of 64 pixel CMOS-integrated FBAR devices with the area less than $1 \mathrm{~mm} \times 1 \mathrm{~mm}$ (Nirschl et al. 2010). The miniaturization of the full system also facilitates to reduce the specimen consumption. This means that each blood test requires a smaller pinprick and hurts less. In this work, the FBAR sensor has the area of only $500 \mu \mathrm{m} \times 500 \mu \mathrm{m}$ (not including the liquid pool) and the sample consumption of $1 \mu \mathrm{l}$, which is much less than that of the existing coagulometer, such as TEG, QCM, turbidimetric and mechanical meter (Harris et al. 2013). In the further work, the existing miniaturized and automatic blood reprocessing module and electronic components can be integrated in the FBAR based system. The cost per test of the FBAR sensor is estimated to be less than 3 dollars considering the equipment depreciation, the fabrication cost of the disposable FBAR devices and the low consumption of the test reagents. This test cost is equivalent to the wildly used paramagnetic particle methods and optical methods in laboratories. However, the 
FBAR based system is more suitable for the personal health monitoring thanks to its compactness, portability, quick readout and simple operation.

\section{Conclusions}

We have demonstrated that the MEMS based lateral field excited FBAR is a feasible tool for studying the rheology of blood. The FBAR working under shear mode was characterized by controlled glycerin solutions to map the frequency-viscosity relation and then used to monitor the coagulation process of the plasma and whole-blood samples. The frequency response had an apparent step-ladder curve that was distinctly suitable for determining the coagulation time. The procoagulant activity of thromboplastin on the coagulation process were obvious. The testing results showed a good consistency between the FBAR sensor and a commercial coagulometer.

Significantly, we were able to employ a small size, light weight, low cost and simple operation to realize the blood coagulation. Only one microliter of blood sample was required for the proposed sensor. The statistical data has proven the precision of the sensor for clinical application. Furthermore, with the integration of MEMS manufacturing techniques, the FBAR sensor offers a high potential for developing miniaturized, completely online and automated analytical system for routine diagnostics of hemostatic status in surgical operations and personal health monitoring. In our further study, we will conduct measurement series toward using pathological and clinically relevant blood samples. 


\section{Acknowledgements}

This work was supported by National Science Foundations of China (No. 61574086,

No. 11504207), National Science Foundation of Shandong Province No. ZR2014AQ006, Qingdao science and technology program of basic research projects [15-9-1-78-jch], Project of Shandong Province Higher Educational Science and Technology Program [J15LJ0]. The authors acknowledge the clinical laboratory of Huangdao district hospital, Qingdao, China for the blood sample collecting.

\section{References}

Andersson, M., Andersson, J., Sellborn, A., Berglin, M., Nilsson, B., Elwing, H., 2005. Quartz crystal microbalance-with dissipation monitoring (QCM-D) for real time measurements of blood coagulation density and immune complement activation on artificial surfaces. Biosens. Bioelectron. 21(1), 79-86.

Anja, B., Søren, D., Stephan Sylvest, K., Silvan, S., Maria, T., 2011. Cantilever-like micromechanical sensors. Rep. Prog. Phys. 74(3), 036101.

Arlett, J.L., Myers, E.B., Roukes, M.L., 2011. Comparative advantages of mechanical biosensors. Nature Nanotechnology 6(4), 203-215.

Beckera, B., Cooper, M.A., 2011. A survey of the 2006-2009 quartz crystal microbalance biosensor literature. J. Mol. Recognit. 24(5), 754-787.

Blombäck, B., Carlsson, K., Fatah, K., Hessel, B., Procyk, R., 1994. Fibrin in human plasma: Gel architectures governed by rate and nature of fibrinogen activation. Thromb. Res. 75(5), 521-538. 
Cao, L., Chang, M., Lee, C.Y., Castner, D.G., Sukavaneshvar, S., Ratner, B.D., Horbett, T.A., 2007. Plasma-deposited tetraglyme surfaces greatly reduce total blood protein adsorption, contact activation, platelet adhesion, platelet procoagulant activity, and in vitro thrombus deposition. Journal of Biomedical Materials Research - Part A 81(4), 827-837.

Chang, H.C., Cheng, T.J., Wu, T.H., Lin, T.M., 2000. Determination of coagulation time in whole blood containing anticoagulant by piezoelectric quartz crystal sensor. Sens. Actuator. B 66(1), 296-298.

Chen, D., Wang, J., Li, D., Xu, Y., Li, Z., 2011a. Solidly mounted resonators operated in thickness shear mode based on c-axis oriented AlN films. Sensors and Actuators A $165,379-384$.

Chen, D., Wang, J., Xu, Y., Li, D., Zhang, L., Liu, W., 2013. A lateral field excited $\mathrm{ZnO}$ film bulk acoustic wave sensor working in viscous environments. J Micromech Microeng 23, 095032-095038.

Chen, D., Wang, J.J., Liu, Q.X., Xu, Y., Li, D.H., Liu, Y.J., 2011b. Highly sensitive $\mathrm{ZnO}$ thin film bulk acoustic resonator for hydrogen detection. J Micromech Microeng 21(11), 1150181-1150187.

Cheng, T.J., Chang, H.C., Lin, T.M., 1998. A piezoelectric quartz crystal sensor for the determination of coagulation time in plasma and whole blood. Biosens. Bioelectron. 13(2), 147-156.

Duner, G., Thormann, E., Dedinaite, A., 2013. Quartz Crystal Microbalance with Dissipation (QCM-D) studies of the viscoelastic response from a continuously 
growing grafted polyelectrolyte layer. J. Colloid Interface Sci. 408, 229-234.

Efremov, V., Killard, A.J., Byrne, B., Lakshmanan, R.S., 2013. The modelling of blood coagulation using the quartz crystal microbalance. J. Biomech. 46(3), 437-442.

Efremov, V., Lakshmanan, R.S., Byrne, B., Cullen, S.M., Killard, A.J., 2014. Modelling of blood component flexibility using quartz crystal microbalance. Journal of Biorheology 28(1), 45-54.

Eom, K., Park, H.S., Yoon, D.S., Kwon, T., 2011. Nanomechanical resonators and their applications in biological/chemical detection: Nanomechanics principles. Phys. Rep. 503(4-5), 115-163.

Evans, P.A., Hawkins, K., Lawrence, M., Williams, R.L., Barrow, M.S., Thirumalai, N., Williams, P.R., 2008. Rheometry and associated techniques for blood coagulation studies. Med. Eng. Phys. 30(6), 671-679.

Fcrre-Pikal, E.S., Dclgado Arámburo, M.C., Walls, F.L., Lakin, K.M., 2001. 1/f frequency noise of 2-GHZ high-Q thin-film sapphire resonators. IEEE T Ultrason Free 48(2), 506-510.

Flewitt, A.J., Luo, J.K., Fu, Y.Q., Garcia-Gancedo, L., Du, X.Y., Lu, J.R., Zhao, X.B., Iborra, E., Ramos, M., Milne, W.I., 2014. ZnO based SAW and FBAR devices for bio-sensing applications. J Non-Newton Fluid 222, 209-216.

Flewitt, A.J., Luo, J.K., Fu, Y.Q., Garcia-Gancedo, L., Du, X.Y., Lu, J.R., Zhao, X.B., Iborra, E., Ramos, M., Milne, W.I., 2015. ZnO based SAW and FBAR devices for bio-sensing applications. J Non-Newton Fluid 222, 209-216.

Freedman, M.D., 1992. Oral Anticoagulants: Pharmacodynamics, Clinical Indications 
and Adverse Effects. J Clin Pharmacol 32(3), 196-209.

Harris, L.F., Castro-López, V., Killard, A.J., 2013. Coagulation monitoring devices: Past, present, and future at the point of care. TrAC, Trends Anal. Chem. 50, 85-95.

Hussain, M., Northoff, H., Gehring, F.K., 2015. QCM-D providing new horizon in the domain of sensitivity range and information for haemostasis of human plasma. Biosens. Bioelectron. 66, 579-584.

Jain, A., Graveline, A., Waterhouse, A., Vernet, A., Flaumenhaft, R., Ingber, D.E., 2016. A shear gradient-activated microfluidic device for automated monitoring of whole blood haemostasis and platelet function. Nature Communications 7.

Jin, J., Jiang, W., Yin, J., Ji, X., Stagnaro, P., 2013. Plasma proteins adsorption mechanism on polyethylene-grafted poly(ethylene glycol) surface by quartz crystal microbalance with dissipation. Langmuir 29(22), 6624-6633.

Kaatz, S., 2010. Impact on patient care: patient case through the continuum of care. J. Thromb. Thrombolysis 29(2), 167-170.

Keiji Kanazawa, K., Gordon Ii, J.G., 1985. The oscillation frequency of a quartz resonator in contact with liquid. Anal. Chim. Acta 175(0), 99-105.

Lakshmanan, R.S., Efremov, V., Cullen, S.M., Killard, A.J., 2014. Measurement of the evolution of rigid and viscoelastic mass contributions from fibrin network formation during plasma coagulation using quartz crystal microbalance. Sens. Actuator. B 192, 23-28.

Lakshmanan, R.S., Efremov, V., O’Donnell, J.S., Killard, A.J., 2016. Measurement of the viscoelastic properties of blood plasma clot formation in response to tissue factor 
concentration-dependent activation. Anal. Bioanal. Chem. 408(24), 6581-6588.

Lee, S., Mortazawi, A., 2016. An Intrinsically Switchable Ladder-Type Ferroelectric BST-on-Si Composite FBAR Filter. IEEE T Ultrason Free 63(3), 456-462.

Lu, Y., Chang, Y., Tang, N., Qu, H., Liu, J., Pang, W., Zhang, H., Zhang, D., Duan, X., 2015. Detection of Volatile Organic Compounds Using Microfabricated Resonator Array Functionalized with Supramolecular Monolayers. ACS Appl Mater Interfaces 7(32), 17893-17903.

Müller, L., Sinn, S., Drechsel, H., Ziegler, C., Wendel, H.P., Northoff, H., Gehring, F.K., 2010. Investigation of prothrombin time in human whole-blood samples with a quartz crystal biosensor. Anal. Chem. 82(2), 658-663.

Martin, S.J., Frye, G.C., Ricco, A.J., Senturia, S.D., 1993. Effect of surface roughness on the response of thickness-shear mode resonators in liquids. Anal. Chem. 65(20), 2910-2922.

Nirschl, M., Rantala, A., Tukkiniemi, K., Auer, S., Hellgren, A.C., Pitzer, D., Schreiter, M., Vikholm-Lundin, I., 2010. CMOS-integrated film bulk acoustic resonators for label-free biosensing. Sensors 10(5), 4180-4193.

Pang, W., Zhao, H., Kim, E.S., Zhang, H., Yuc, H., Hu, X., 2012. Piezoelectric microelectromechanical resonant sensors for chemical and biological detection. Lab on a Chip 12, 29-44.

Parlak, Z., Biet, C., Zauscher, S., 2013. Decoupling mass adsorption from fluid viscosity and density in quartz crystal microbalance measurements using normalized conductance modeling. Meas. Sci. Technol. 24(8). 
Rodriguez-Pardo, L., Fariña, J., Gabrielli, C., Perrot, H., Brendel, R., 2004. Resolution in quartz crystal oscillator circuits for high sensitivity microbalance sensors in damping media. Sens. Actuator. B 103(1-2), 318-324.

Ruby, R., Small, M., Bi, F., Lee, D., Callaghan, L., Parker, R., Ortiz, S., 2012. Positioning FBAR technology in the frequency and timing domain. IEEE T Ultrason Free 59(3), 334-345.

Vikinge, T.P., Hansson, K.M., Benesch, J., Johansen, K., Rånby, M., Lindahl, T.L., Liedberg, B., Lundstöm, I., Tengvall, P., 2000a. Blood plasma coagulation studied by surface plasmon resonance. Journal of Biomedical Optics 5(1), 51-55.

Vikinge, T.P., Hansson, K.M., Sandström, P., Liedberg, B., Lindahl, T.L., Lundström, I., Tengvall, P., Höök, F., 2000b. Comparison of surface plasmon resonance and quartz crystal microbalance in the study of whole blood and plasma coagulation. Biosens. Bioelectron. 15(11-12), 605-613.

Voiculescu, I., Nordin, A.N., 2012. Acoustic wave based MEMS devices for biosensing applications. Biosens. Bioelectron. 33(1), 1-9.

Waggoner, P.S., Craighead, H.G., 2007. Micro- and nanomechanical sensors for environmental, chemical, and biological detection. Lab on a Chip - Miniaturisation for Chemistry and Biology 7(10), 1238-1255.

Wang, J., Chenb, D., Xub, Y., Liu, W., 2014. Label-free immunosensor based on micromachined bulk acoustic resonator for the detection of trace pesticide residues. Sens. Actuator. B 190, 378-383.

Wingqvist, G., 2010. AlN-based sputter-deposited shear mode thin film bulk acoustic 
resonator (FBAR) for biosensor applications - A review. Surf. Coat. Technol. 205(5), $1279-1286$.

Wu, H., Zhao, G., Zu, H., Wang, James H.C., Wang, Q.-M., 2016. Real-Time Monitoring of Platelet Activation Using Quartz Thickness-Shear Mode Resonator Sensors. Biophys. J. 110(3), 669-679.

Xu, W.C., Appel, J., Chae, J., 2012. Real-Time Monitoring of Whole Blood Coagulation Using a Microfabricated Contour-Mode Film Bulk Acoustic Resonator. J Microelectromech S 21(2), 302-307.

Zinck, C., Emmanuel, D., Volatier, A., Caruyer, G., Tanon, D.P., Figuiere, L., 2005. Design, integration and characterization of PZT tunable FBAR. IEEE International Symposium on Applications of Ferroelectrics, pp. 29-32. 


\section{List of figure captions}

Fig. 1 The basic device configuration and the testing system of the FBAR sensor for blood coagulation monitoring. (a) The microphotograph and the cross-section view of the FBAR device; (b) the system diagram; (c) the packaged PCB of the testing system; (d) the photograph of overall system

Fig. 2 The resonant frequency of the lateral field excited FBAR device as the function of the value of $\sqrt{\rho_{l} \eta_{l}}$ following in aqueous glycerin solutions. The error bars are standard deviation of the ten times measurements. The frequency difference of the reference device and the detector device is used as the response.

Fig. 3 The time-dependent resonant frequency profiles in the absence and presence of increasing concentration of TP in the blood samples. (a) The plasma samples; (b) the whole-blood samples.

Fig. 4 (a) The correlation of PT values determined by the commercial coagulometry and FBAR sensor. (b) The Bland-Altman plot of the FBAR sensor and the commercial coagulometer. The error bar of the coagulation time is the standard deviation of ten times measurements for each blood sample.

Fig. 5 The time-dependent resonant frequency profiles of the plasma samples mixed with different concentrations of heparin. (a) no heparin; (b) $0.2 \mathrm{IU} / \mathrm{ml}$; (c) 0.4 IU/ml; (d) $1 \mathrm{IU} / \mathrm{ml}$. The plasma samples were activated by $50 \mathrm{unit} / \mathrm{ml} \mathrm{TP}$ solution. 


\section{Tables}

Table 1 The measured frequency shift and the PT values of the FBAR sensor for different plasma and whole-blood samples.

\begin{tabular}{|c|c|c|c|c|c|c|c|}
\hline & & \multicolumn{3}{|c|}{ Plasma samples } & \multicolumn{3}{|c|}{ Whole-blood samples } \\
\hline Experiment & $\begin{array}{c}\mathrm{TP} \\
\text { concentration } \\
\text { (unit/ml) }\end{array}$ & $\begin{array}{c}\text { Frequency } \\
\text { decrease* }^{*} \\
(\mathrm{MHz})\end{array}$ & $\begin{array}{c}\text { PT } \\
(\mathrm{Sec})\end{array}$ & C.V** & $\begin{array}{c}\text { Frequency } \\
\text { decrease* } \\
(\mathrm{MHz})\end{array}$ & $\begin{array}{c}\text { PT } \\
(\mathrm{Sec})\end{array}$ & C.V** \\
\hline 1 & 0 & -- & $\begin{array}{c}\text { No } \\
\text { coagulation }\end{array}$ & -- & -- & $\begin{array}{c}\text { No } \\
\text { coagulation }\end{array}$ & -- \\
\hline 2 & 1 & $-8.971 \pm 0.13$ & $682 \pm 16$ & $3.6 \%$ & $8.714 \pm 0.14$ & $722 \pm 24$ & $2.8 \%$ \\
\hline 3 & 10 & $-8.951 \pm 0.10$ & $548 \pm 15$ & $2.4 \%$ & $8.821 \pm 0.13$ & $565 \pm 17$ & $2.6 \%$ \\
\hline 4 & 50 & $-8.386 \pm 0.15$ & $232 \pm 6$ & $2.5 \%$ & $8.759 \pm 0.16$ & $262 \pm 8$ & $3.2 \%$ \\
\hline 5 & 100 & $-8.177 \pm 0.16$ & $130 \pm 4$ & $3.0 \%$ & $8.715 \pm 0.12$ & $168 \pm 5$ & $2.3 \%$ \\
\hline
\end{tabular}

$* 95 \%$ of the maximum downshift of frequency $(\Delta f)$

** The coefficient of variation between the results of FBAR sensor and the commercial coagulometer. 

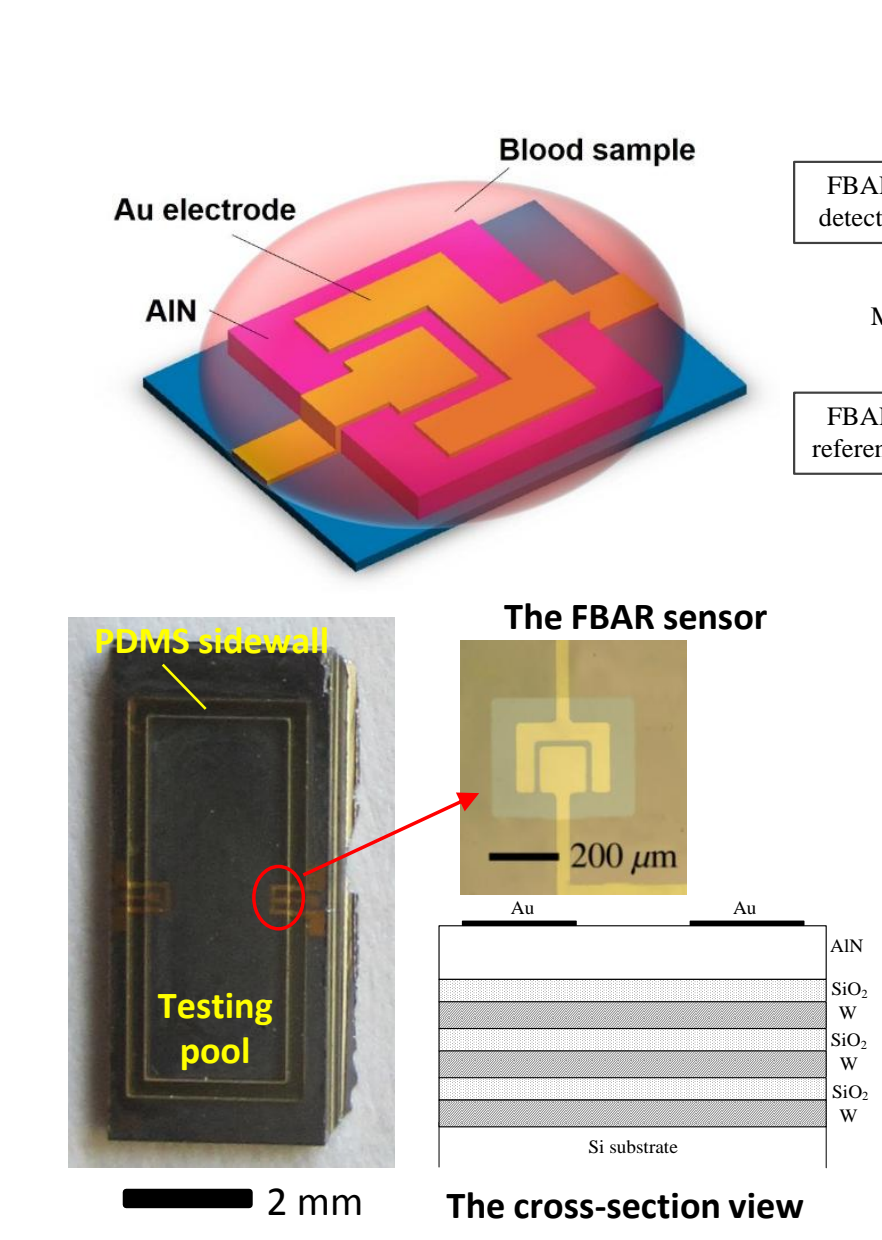

(a)

(a)

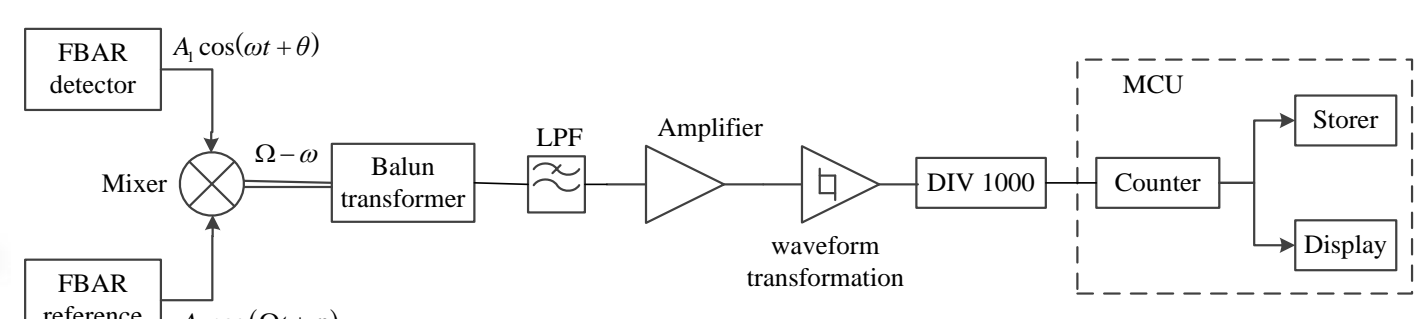

(b)

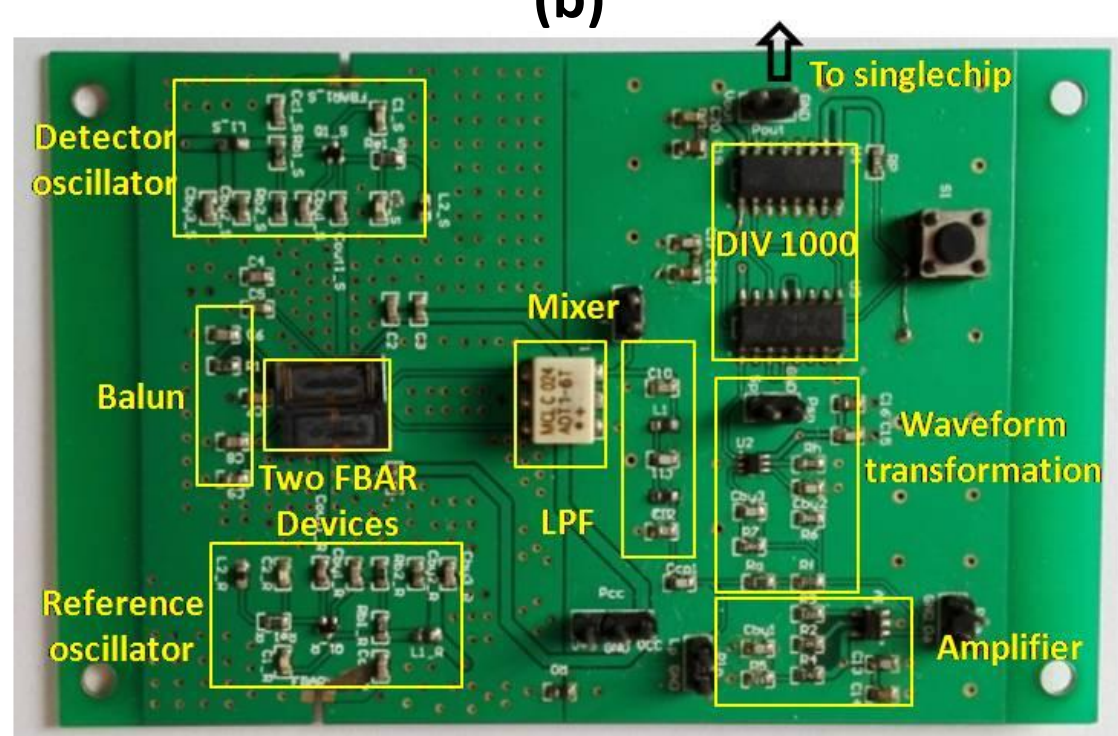

(c)
)
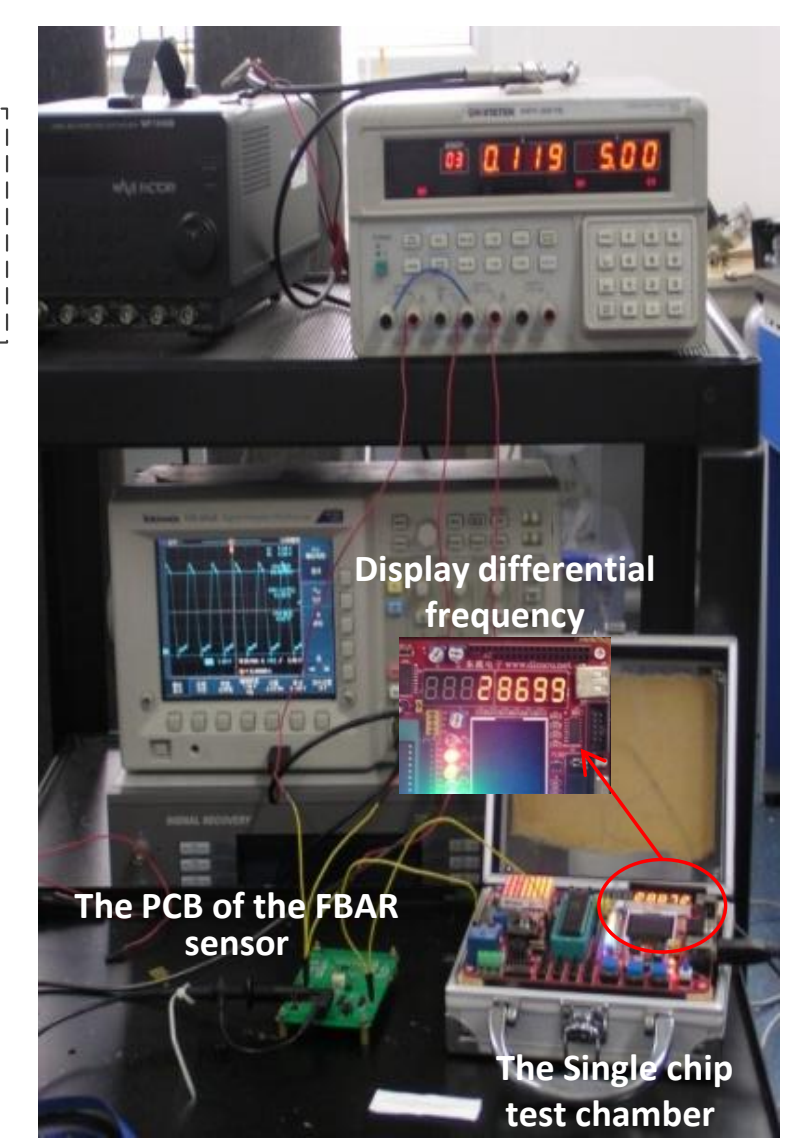

(d) 


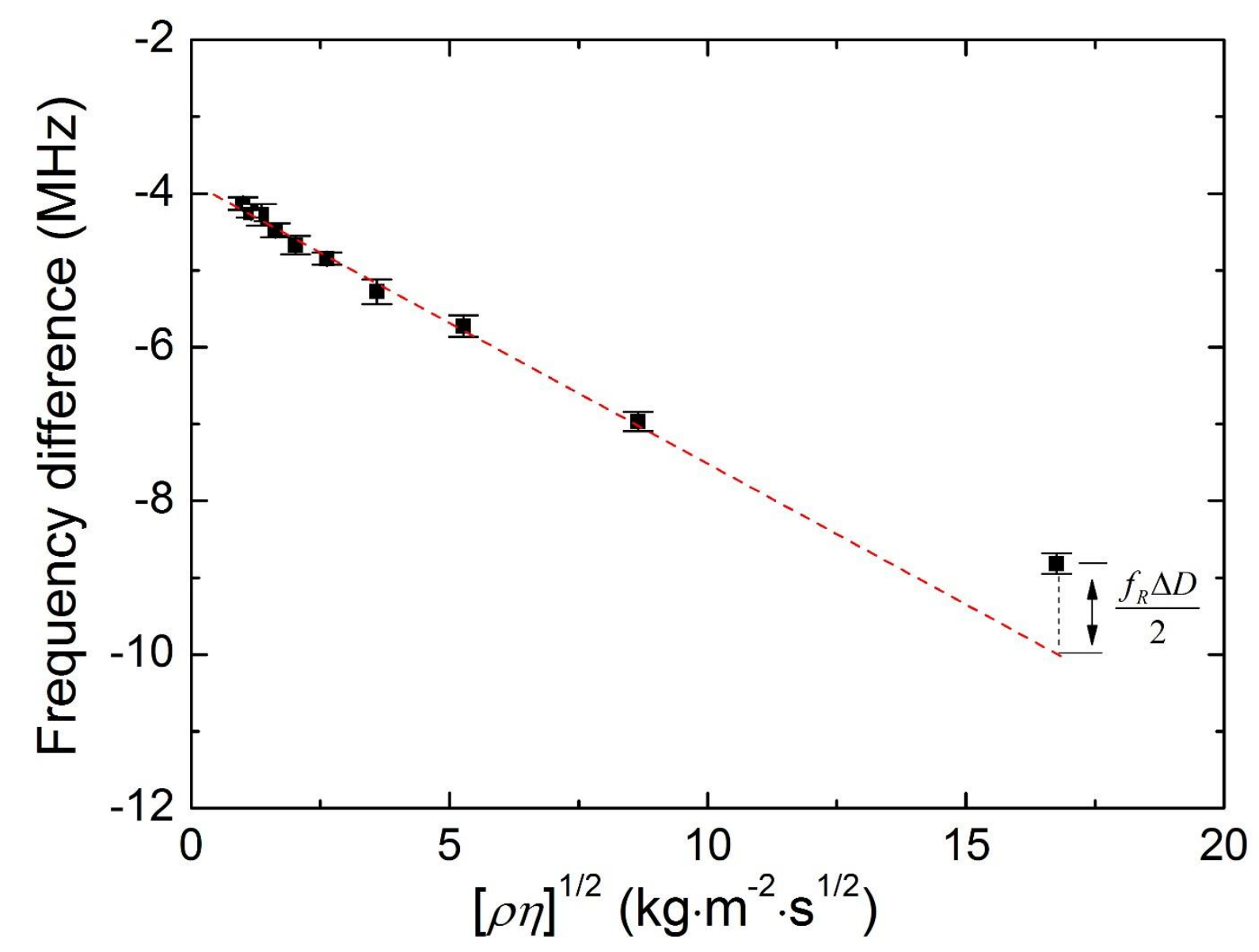

$[\rho \eta]^{1 / 2}\left(\mathrm{~kg} \cdot \mathrm{m}^{-2} \cdot \mathrm{s}^{1 / 2}\right)$ 

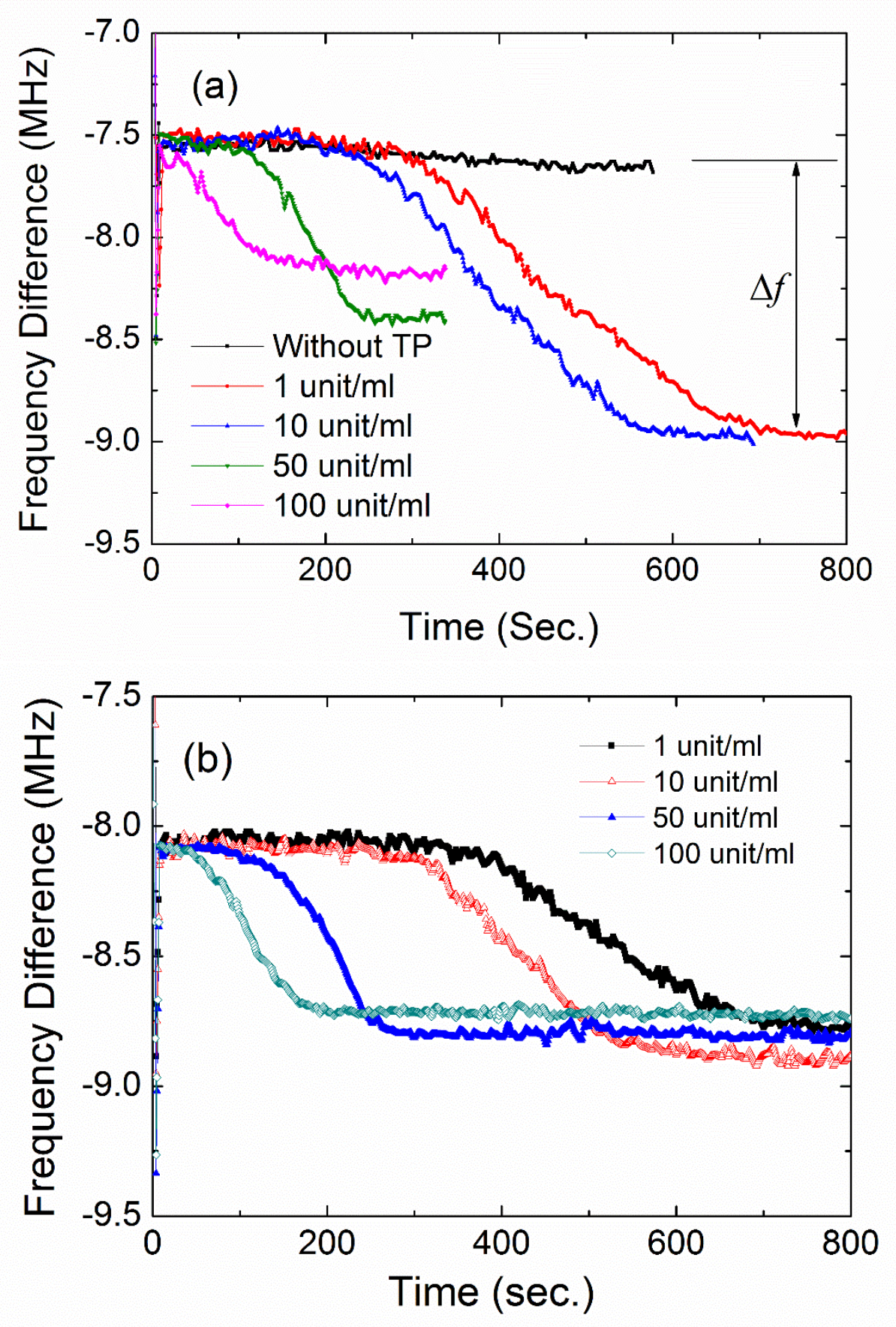

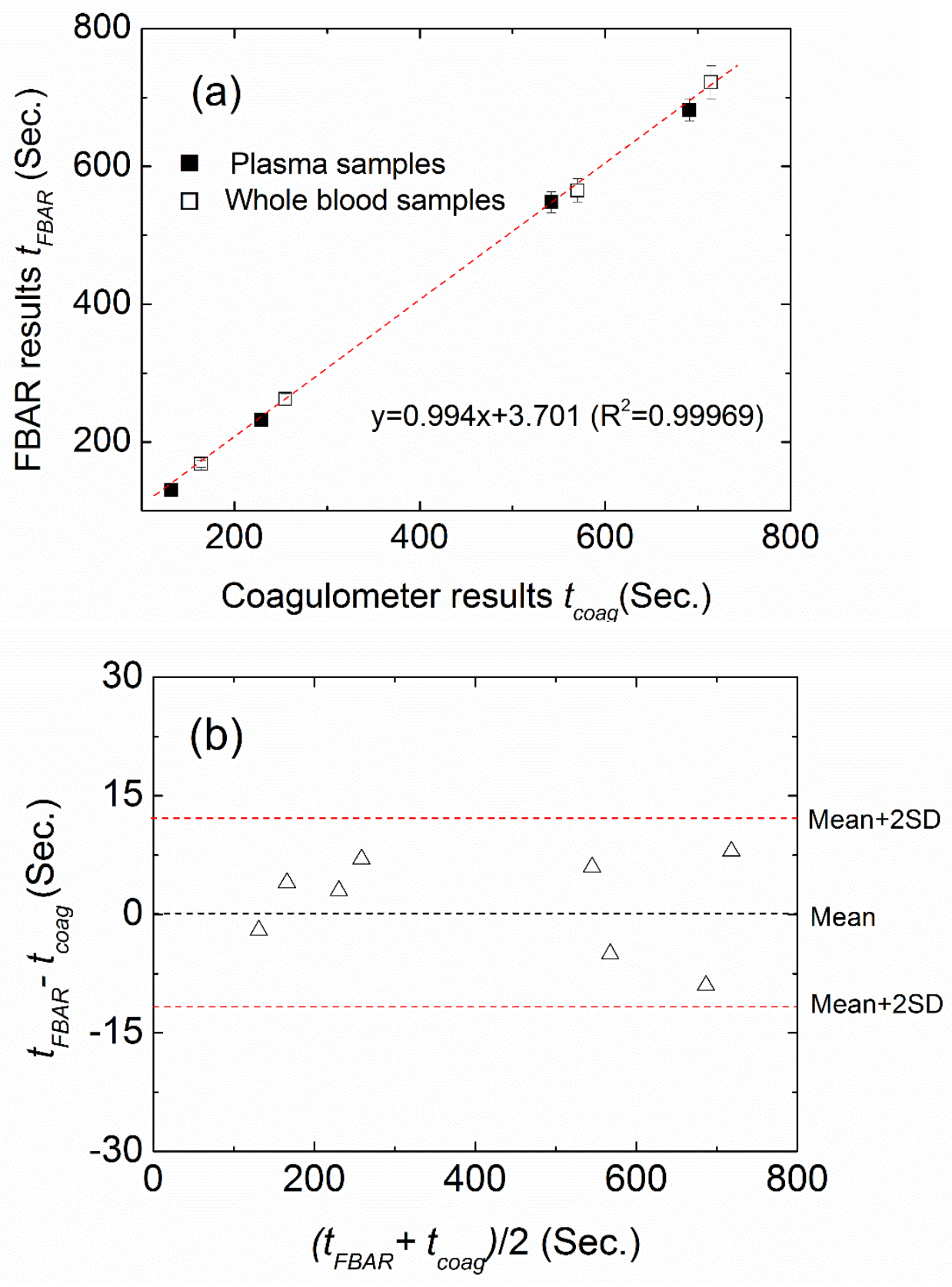
